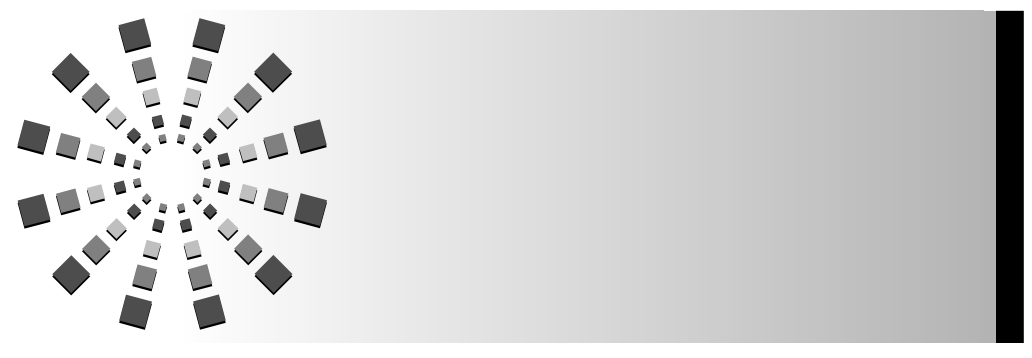

Ruth McConigley, PhD, RN

Kristi Holloway, BSc (Hons), RN

Jodi Smith, Grad Dip Gerontology, OT

Georgia Halkett, PhD, FIR

Jo Keyser, PGrad Dip Hlth Ed, RN, SBN

Samar Aoun, PhD

Leanne Monterosso, PhD, RN, RM, NNT, FRCNA

\title{
The Diagnosis and Treatment Decisions of Cancer Patients in Rural Western Australia
}

\section{$\begin{array}{llllllll}\text { K } & E & \text { Y } & \text { W } & \text { O } & R & \text { D } & S\end{array}$}

Cancer diagnosis

Cancer treatment

Decision making

Rural
Background: People living in rural areas who have a diagnosis of cancer have poorer outcomes than people living in urban centers. The reasons for this are unclear. Little is known about the impact that living in a rural area has on the diagnosis and treatment decisions of these people and how these may in turn impact on care outcomes. Objectives: This study explored the reasons why people living in rural areas may delay diagnosis and what issues affected the decisions they made regarding their cancer treatment. Methods: In depth, semistructured interviews were conducted with 18 participants from 3 rural Western Australian health regions. Content analysis was used to develop themes. Results: Four themes were identified to describe the rural cancer experience. The first 3 themes, Experiences of Diagnosis and Referral, The Treatment Journey, and Managing your own Care, relate to the experiences of rural cancer patients during their journey through the health care system. The final overarching theme, Implicit Faith, described the level of confidence that rural cancer patients had in the health system, often despite delays and inconveniences. Conclusions: There is a need to improve primary health care and care coordination for rural cancer patients living in Australia and to promote self-advocacy and consumer empowerment for rural cancer patients. Implications for Practice: Rural patients need help and support throughout their cancer journey, including through the process of diagnosis. ortality rates for people with a diagnosis of cancer in rural Australia are significantly higher compared with rates in urban areas. ${ }^{1}$ In particular, rural people with a diagnosis of lung, colorectal, or prostate cancer are $10 \%$ to $20 \%$ more likely to die of their disease than people living in urban areas. Reasons for these discrepancies are
Author Affiliations: Western Australian Centre for Cancer and Palliative Care, Curtin Health Innovation Research Institute, Curtin University, Perth (Dr McConigley, Mss Holloway and Smith; and Drs Halkett, Aoun, and Monterosso); and Western Australian Country Health Service, Midwest and Murchison Region (Ms Keyser), Western Australia.

This project was funded by the WA Cancer and Palliative Care Network Small Grants 2009.
Correspondence: Ruth McConigley, PhD, RN, Western Australian Centre for Cancer and Palliative Care, Health Research Campus, Curtin University, GPO Box U1987, Perth, Western Australia 6845 (R.McConigley@ curtin.edu.au).

Accepted for publication August 12, 2010.

DOI: 10.1097/NCC.0b013e3181f795e7 
unclear. However, rural areas in Western Australia (WA) have fewer primary health services than urban areas in WA. In particular, there are fewer general practitioners (GPs), limited access to diagnostic services such as magnetic resonance imaging, limited access to surgeons and medical oncologists, and limited access to chemotherapy. ${ }^{2}$ Furthermore, radiotherapy is available only in the Perth metropolitan area. The lack of such vital cancer care services is likely to have a negative impact on survival, and as remoteness increases, so too does the probability of dying of cancer. ${ }^{3}$ At present, the WA Department of Health is undertaking significant restructuring of cancer services throughout the state. In rural areas, in an attempt to address the disparity in cancer outcomes, a range of measures is being implemented including the development of regional cancer centers, multidisciplinary cancer teams, and increasing access to oncology specialists in rural centers. Rural cancer nurse coordinators (RCNCs) have been providing care coordination and support services in rural WA since $2007 .{ }^{4}$

Delays in cancer diagnosis are linked with higher cancer morbidity and mortality. ${ }^{5,6}$ International studies have shown that when compared with their urban counterparts, rural and remote people tend to present to a medical practitioner later in their cancer trajectory. ${ }^{7-9}$ Factors cited as affecting the speed of diagnosis for rural people with cancer include comorbidities that make early identification of symptoms difficult, seasonal work leading to delays in presenting for medical attention, the impact of informal social networks on health decision making, and difficultly in scheduling medical appointments. ${ }^{10}$ Therefore, people living in rural areas may have a cancer journey that differs from people living in urban areas.

A number of studies internationally have found that people living in rural areas receive treatment for their cancer that is less than optimal. ${ }^{11-14}$ Traveling to receive treatment is often required by rural and remote people with cancer, and this can be stressful and expensive. ${ }^{15}$ Financial concerns are also reported to affect rural residents' decisions about cancer care more than their metropolitan counterparts. ${ }^{16}$ Several authors have also suggested that rural people are more likely to refuse treatment than people living in metropolitan centers. ${ }^{10,17-19}$

It is important the impact of geographic location on diagnosis and treatment decisions made by rural cancer patients is investigated. This information will provide the evidence on which to base interventions that target provision of ruralspecific practical support and assistance.

This project aimed to explore the decision making of rural people with a diagnosis of cancer. More specifically, the project sought to answer the following questions:

1. Do rural people delay seeking medical attention for suspicious symptoms that could be linked to cancer?

2. Do people with cancer living in rural locations in WA make treatment decisions other than those recommended by their GP?

3. How does location affect decisions made by rural people with a diagnosis of cancer?

4. What issues, other than location, affect decision making about cancer treatment?
5. What support services or information is required to assist rural people when making treatment decisions about cancer?

\section{Methods}

\section{Ethics}

Approval to conduct this study was granted by the human research ethics committees of Curtin University of Technology (HR 99/2009) and the Western Australian Country Health Service (2009:16).

\section{Design}

A descriptive, exploratory qualitative design was used to explore the experiences of rural cancer patients. This type of method allows for a broad exploration of a topic, about which little is currently known. ${ }^{20}$

\section{Setting}

Participants were recruited from 3 health regions in WA: the Great Southern, which is the most southern region in WA; the Wheatbelt, which surrounds the northern and eastern parts of Perth's metropolitan boundary; and the Midwest region, which covers the central west of the state. These areas were chosen because they represent a cross section of Western Australian rural communities including agricultural, coastal retirement, mining, and remote communities and a variety of rural settings described by the Rural, Remote, and Metropolitan Area Classifications ${ }^{21}$ as ranging from zone 4 (small rural center) to zone 7 (remote center). Each region has in excess of 250 people with a diagnosis of cancer each year. ${ }^{22}$

\section{Sample}

Purposive sampling was used to identify participants diagnosed with cancer in 2008 or 2009 , with a range of cancer diagnoses and cancer experiences including having treatment in the nearest regional center or traveling to an urban center for treatment.

\section{Inclusion and Exclusion Criteria}

Subjects were included if they were older than 18 years, English speaking, able to provide written informed consent, at least 3 months postdiagnosis, and considered well enough to participate in interviews by the cancer team in each region.

Subjects were excluded if they had any cognitive deficits or communication difficulties.

\section{Procedure}

Eligible participants were identified by RCNCs or the palliative care coordinator who introduced the study and ascertained potential participants' suitability and interest in participating. People who were interested in the study were sent an information pack by the research assistant (J.S.) that included 
a study information sheet, consent form, and a stamped, addressed return envelope. They were asked to return the signed consent form if they wished to participate. On receipt of the consent form, potential participants were contacted by the research assistant to arrange an interview.

Semistructured interviews were conducted either face-toface or on the telephone, depending on participant location, participant choice, and accessibility. The interviewer (J.S.) was a health professional with experience in research interviewing. Time was spent with participants prior to interview commencement to develop rapport and enhance the quality of data collected. An interview guide included questions aimed at identifying key issues that most influenced decisions relating to diagnosis and treatment, including choice of treatment location (city or rural area), the type of treatment chosen, information provided to help inform decisions and practical needs (see Appendix 1). An event history calendar was used to measure delay in seeking diagnosis and treatment for suggestive symptoms. ${ }^{23}$ This technique aimed to reduce recall bias by relating patient experiences to memorable dates in the previous year. $^{24}$

\section{Data Analysis}

Data were managed using NVivo 8 software (QRS International, Cambridge, MA). Key concepts that affected treatment decision making of rural cancer patients were identified using inductive content analysis. ${ }^{25}$ Each transcript was read and analyzed independently by 2 researchers (R.M., K.H.), and intercoder agreement was determined to ensure a similar understanding of concepts and developing categories. ${ }^{26}$ Consensus was then reached between researchers about each concept, and then about each category as they developed. Categories were developed to describe the experiences of cancer patients living in rural WA. Rigor of the analysis was ensured by applying the criteria of credibility, auditability, neutrality, and fittingness. ${ }^{27}$ Credibility was maintained by the researchers completing independent coding and the use of member checks with participants and 1 RCNC (J.K.), to ensure that categories accurately captured issues being discussed. Auditability was demonstrated by keeping memos to document all theoretical decision making. Neutrality was addressed by having 2 researchers independently analyze the data. Inclusion of participants from different regions with varying backgrounds and experience helped to establish the transferability of findings.

\section{- Results}

In total, 20 people were approached to participate; 1 refused, 1 died prior to participation, and 18 interviews were conducted. Fourteen of the interviews were conducted face-toface; the other 4 were conducted via telephone. Interviews lasted between 10 and 90 minutes. Two interviews were of short duration (10 and 15 minutes); however, these participants had specific dates and points written down that they wished to discuss, and therefore, their transcripts yielded rich data. The demographic profile of the participants is included in the Table. Most participants were male $(\mathrm{n}=12,66.6 \%)$ and married $(\mathrm{n}=11,61.1 \%)$. The mean age of participants was $57.6(\mathrm{SD}, 14.8)$ years, and the majority lived in small rural centers (Rural, Remote, and Metropolitan Area Classifications zone 4) $(n=10,55.5 \%)$. Despite living in a rural center, access to cancer services and facilities was limited. The most common cancer diagnoses were lung cancer $(\mathrm{n}=5$, $27.8 \%)$. followed by bowel cancer $(\mathrm{n}=4,22.2 \%)$.

Four main themes emerged from the data. The first 3 themes, Experiences of Diagnosis and Referral, The Treatment Journey, and Managing your own Care, related to the experiences of rural cancer patients during the period of diagnosis followed by the journey through cancer treatment. The final and overarching theme, Implicit Faith, describes the positive attitude patients had toward their cancer care, despite their accounts that suggest that they experienced delays and inconveniences.

\section{Experiences of Diagnosis and Referral}

Delays in seeking and receiving a diagnosis were common, with a variety of issues related to these delays. Some participants did not recognize symptoms as warranting attention or delayed seeking medical advice about symptoms that were causing them discomfort because of other obligations. One participant explained:

Well I just knew something was wrong with the waterworks, you know... And I didn't get diagnosed for another year. What did I do? I ignored it. No, well

\section{*: Table $\cdot$ Demographic Details of Participants $(n=18)$}

\begin{tabular}{|lc}
\hline & $\mathrm{n}(\%)$ \\
\hline Sex & \\
Male & $12(66.6)$ \\
Female & $6(33.3)$ \\
Age, $y$ & \\
$31-40$ & $3(16.7)$ \\
$41-50$ & $2(11.1)$ \\
$51-60$ & $4(22.2)$ \\
$61-70$ & $6(33.3)$ \\
$71-80$ & $1(5.5)$ \\
$80+$ & $2(11.1)$ \\
Marital status & \\
Married & $11(61.1)$ \\
Single/divorced & $7(38.9)$ \\
Cancer diagnosis & \\
Lung & $5(27.8)$ \\
Bowel cancer & $4(22.2)$ \\
Breast & $2(11.1)$ \\
Gynecologic & $2(11.1)$ \\
Other & $5(27.8)$ \\
Location (RRMA classification) & \\
Small rural center (zone 4) & $10(55.5)$ \\
Rural center (zone 5) & $5(27.8)$ \\
Remote center (zone 6) & $1(5.5)$ \\
Remote area (zone 7) & $2(11.1)$ \\
&
\end{tabular}

Abbreviation: RRMA, Rural, Remote, and Metropolitan Area Classifications. 
I did that I suppose, but I had things to do and I just kept at what I was doing. I knew something was wrong, but I blew it. (Interview 5)

For some participants seeking medical advice led to a different diagnosis, which delayed the diagnosis of cancer, or their symptoms were not given immediate attention by their GP. Participants who presented to their GP with a symptom that was worrying them were not always referred for testing immediately, often because symptoms were nonspecific, or they were not compliant with GP suggestions. One participant described her experience:

I could feel a lump on the side of my breast, probably about the size of a small ball bearing or a big ball bearing. Not as big as a pea though. I went to my doctor one time, and he couldn't feel it so he said to me come back at a different time of the month when it might... he might be able to feel it better. But I don't go to the doctor very often, so it was 12 months later when I went. (Interview 2)

Other participants delayed seeking advice until symptoms were so acute that they required immediate care in a hospital emergency department. One participant discussed how he had been having difficulty urinating for months and disregarded the symptoms until they were so acute that he could no longer ignore them:

Well I got up in the morning, it was about, about 2 o'clock in the morning. Had to go for a pee. I started peeing, and it was very hard and, and I, and I noticed there was blood coming out, and I thought, "Oh, you know, there's something wrong here." ...And there were clots coming out, and that scared me and that's when I said to the wife, "There's something wrong, and can you take me to the hospital?" That's how I found out. (Interview 12)

The emergency department was described by several participants as a place where care was fast-tracked and so represented a quick way to have suggestive symptoms examined by a medical practitioner.

On diagnosis, referrals to support services were ad hoc and often deferred. For some people, this delay was perceived as critical:

There was a long delay at a crucial time for her with her particular type of cancer at the time so... we didn't know then who to contact and who to see or to just wait and then contact you, but there was no one coordinating that. From where we had the scans and PET [positron emission tomography] scans [in the city], back to your GP and the specialist, and you know they were away on holidays and nobody sort of got onto it. (Interview 17)

Once "in the system," the available support services were considered of great benefit, particularly practical supports such as travel and accommodation subsidy. One participant described his experience:

I just think that, once, once you get into the system and, and kind of realize what support there is and the, and facilities there, and the treatment that's there, yes, it's just been all first rate, really. (Interview 13)

Support from RCNCs was described as helpful in navigating the health system. Participants highlighted the RCNCs as great sources of support, information, advice, and importantly patient advocacy. One participant described how the cancer nurse coordinator liaised between specialists to solve a treatment decision:

And she [RCNC] went and spoke to, you know, like the radiation oncologist who then spoke to the medical oncologist... and we got things sorted out... if it hadn't have been for [the RCNC] we wouldn't have known half the things that was going on. (Interview 13)

Despite the positive impacts of the RCNC role on the participant's experiences, several participants described a delay in being referred to the RCNC and emphasized the referral to RCNCs as essential and needing reform.

\section{The Treatment Journey}

The process of making decisions about treatment options appeared to be a simple one for participants, mainly because they did not appear to question the treatment options recommended by medical specialists. One participant said:

He's [the doctor] the one who's making all the decisions here. And if he thinks it's okay well, good for me too I reckon. (Interview 9)

It also appeared that many patients were not offered options to have care closer to home and were unaware that in many cases they could have some, or all, of their care provided in the local hospital. One participant explained how he began to have his chemotherapy at the regional hospital:

Eventually, the option came up. And then in July, to do chemo... at [the local hospital]... up until then, I had to go to Perth. (Interview 12)

Travel to major treatment centers in the city was described as problematic, but necessary. Participants discussed issues related to the cost of travel, being away from family, and difficulties continuing work throughout treatment. Some participants felt that the travel for treatment was not warranted and too stressful:

Just the journey down from here to [Perth Hospital] and having to sit around all day, and then have 5 hours or 6 hours of chemo. It just got too much, and he [husband] was saying, "Why do we have to do this?" Or "I can't take any more of this." ...It just got too much. (Interview 14)

Those who were offered treatment in the regional areas considered it to be convenient and less stressful:

Well it would be too inconvenient to go to Perth.

... Because as I said, my wife works. ....And that's one of the reasons why they thought, "No it would be better with a doctor up here." ...Saves a lot of hassle, trying to find accommodation. (Interview 12) 
However, not all participants were offered treatment close to home, either because they or their medical team preferred treatment to be provided in an urban-based tertiary hospital, or the treatment required included radiotherapy, which is available only in the metropolitan area.

\section{Managing Your Own Care}

Participants felt that they needed to manage their illness and advocate for themselves to ensure they received the best possible care. One participant described this process:

There was some swelling or inflammation there, and it coupled with it had increased in size, and I said, "Look that is showing, there is some process happening, and I want something else done about it. I want a-," so they sent me off down to Perth for a PET scan. But again, I had to stand up, and you know really put my hand up you know. Yes. I had to, you just got to be your own advocate and insist that something be done. (Interview 13)

Participants also needed to coordinate their own care, in part because communication between rural and city medical professionals was described as limited:

No, the only other thing was that I felt that I've had to keep asking the doctors in Perth to inform my [specialist] and my GP. They tend not to do it unless you really push them to do it. So quite often, I feel that a few people are sort of working in the dark. They've got half the information that I have. I just, you know, if they could just, I think their report, their report and just send it off as automatically it would make a big difference to the, to the coordination. (Interview 4)

Participants who were able to manage their own care, often because of prior experience in the health industry, reported a better experience than those who were less able to advocate on their own behalf.

\section{Implicit Faith}

The overarching theme in this study was that of implicit faith. Participants described incidents relating to their cancer journey that suggested that they had had difficult experiences, such as delayed diagnoses, extensive travel for diagnostic procedures, and cancer treatment and communication difficulties between care providers. Despite this, all participants described their care as being good or excellent and were happy with the care they had received. One participant said:

Yes. But everything was generally pretty good. If there were any other issues, like the pain factor, for example, it was certainly no trouble. All you had to do was go and talk to one of the nurses, particularly in the radiation or talk to one of the girls at the reception in oncology, you know, the radiation, the medical oncology...

(Interview 13)

Even when participants had experienced difficulties with travel and accommodation or delays in seeing the required health professionals, they continued to describe their experiences as good and the health professionals who had cared for them as providing a high standard of care. This faith in the health care system appeared to lead to participants accepting care that was difficult and stressful for them without complaint and without questioning if care could be improved and stressors lessened.

\section{Discussion}

The findings of this study reflect the realities of health care in rural Australia, in that some participants lived in areas that were too remote for the interviewer to travel and conduct a face-to-face interview. As such, interviews were conducted by telephone so that people living in remote areas were able to be included in the sample. There is some discussion in the literature about the appropriateness of conducting research interviews by telephone because of difficulties establishing rapport and the lack of ability to interpret body language. ${ }^{28,29}$ However, in this study, the use of telephone interviewing allowed rural residents who may otherwise have been considered inaccessible because of excessive travel time to conduct the interview to be included in the sample. Other authors have also found telephone interviews to be useful to increase access to otherwise marginalized groups. ${ }^{30,31}$

The findings of this study suggest that rural people may experience delays in having a diagnosis of cancer for a variety of reasons. The reluctance of rural people to seek medical advice has been described by several authors, ${ }^{32,33}$ as has the practice of attending to health care on a seasonal basis to fit with work routines such as harvest. ${ }^{10}$ Addressing these entrenched issues is essential if the rural health differential is to be challenged and care improved. Furthermore, it is possible the fatalism often ascribed to rural people ${ }^{34,35}$ affects the decisions they make about their cancer journey. Participants in this study expressed satisfaction with their care even when they had faced considerable delays or hardships. Similar results were reported by Lamarche and colleagues, ${ }^{36}$ who found the more remote a person's location, the more likely he/she was to express satisfaction with medical care, despite difficulties associated with access to care. Challenging this tolerance of delays and inconveniences associated with rural cancer care and improving rural patients' understandings of their diagnostic and treatment pathway may encourage and empower rural people to expect nothing less than evidence-based, best practice care. This may, in turn, reduce the disparity in outcomes for rural cancer patients.

A study in New Zealand found that $37 \%$ of people with a diagnosis of lung cancer presented to an emergency department with suggestive symptoms rather than presenting to their $\mathrm{GP}^{37}$ and that those people who presented via the emergency department were more likely to have advanced disease. The authors concluded that there were likely to be specific barriers to some groups of people, particularly minority groups, accessing medical care from primary care professionals. It is possible this may also be the case in rural areas, leading to 
some people delaying seeking treatment and subsequently having poorer outcomes. Further research is required to explore potential barriers to seeking assistance in the primary care setting for suggestive or distressing symptoms. As well, initiatives designed to improve primary health in rural areas, such as the introduction of practice nurses and nurse practitioners, must be given attention to reduce the burden on primary health care services and allow greater time and attention for patients presenting for advice regarding symptoms that may be indicative of cancer.

Location did not appear to affect the decisions made by this small group of rural people with a diagnosis of cancer. Participants clearly stated they accepted whatever curative treatment was deemed most appropriate for them by their doctor, despite potential difficulties such as the cost and inconvenience of traveling long distances to treatment centers. However, for some participants, treatment was not completed because of the stress associated with travel and being away from home for extended periods. Overall, it appeared the rural people in this study accepted the inconvenience because it was an expected part of living in a rural area. They were also aware that if they needed health care, it may be difficult to access; however, this was understood and accepted as part of the landscape of choosing to live in the country. This finding was in contrast to several studies that have found that access to high-quality treatment close to home, wherever possible, was the preferred option for most rural people. $^{17,38}$

The findings of this study highlight the importance of cancer nursing roles in rural areas. The role of rural cancer nurses has been established for some time in North America $^{39,40}$ and Great Britain ${ }^{41,42}$ but is relatively new in rural Australia. Research ${ }^{43,44}$ suggests that cancer nurse coordinators and cancer support workers can improve coordination of care for rural people with cancer. A recent literature review ${ }^{45}$ reported that cancer nurses can play an important part in providing information about the cancer journey to patients, thereby empowering patients to make informed decisions about their care. Further evaluation of rural cancer nurse roles would be useful to articulate the scope and potential impact of cancer nurses on rural cancer patient outcomes.

The findings of this study also suggest a need to empower rural cancer patients to take charge of their own care. Unfortunately, because of the distances and isolation associated with the geographic reality of living in rural Australia, cancer patients from more remote areas will always have more limited access to cancer support services than people in more populated areas. However, strategies that promote selfmanagement and self-advocacy (such as the Cancer Resource Kit developed by RCNCs in country $\mathrm{WA}^{46}$ ) may help these patients to navigate the health care system and access care and support appropriate to their needs in a timely manner. Tools to enhance communication between patients and their cancer care team may also be helpful for cancer patients at diagnosis to help them to discuss treatment options with their cancer team. ${ }^{47}$ Further research into promoting self- management and self-advocacy in rural cancer care is warranted to explore the efficacy of these measures in improving patient outcomes.

Use of information and communication technologies such as telehealth were notably absent in most participants' descriptions of their care and could be a useful tool to promote the interface between urban cancer specialists and rural primary health care teams. Telehealth has been well established as a tool for use in enhancing rural health care. ${ }^{48}$ Several authors have demonstrated that the use of telehealth for cancer team meetings was as effective as face-to-face team meetings in terms of decision-making capacity. ${ }^{49,50}$ Expanding the use of technologies to promote care has the potential to promote improved care coordination for rural participants and increase communication between rural primary care teams and urban specialist oncologists.

This study was conducted at a time when several initiatives aimed at addressing the disparate outcomes for rural cancer patients in WA were being implemented. ${ }^{4}$ As such, it would be appropriate to reconsider the diagnosis and treatment decisions and care outcomes for rural cancer patients when these initiatives have been successfully implemented.

\section{- Limitations}

This study used a small sample size $(\mathrm{n}=18)$. Most participants were recruited with the assistance of RCNCs, meaning that only a small number of people who had not received this level of support were included. However, the qualitative methodology applied generated rich data that detail the current role of decision making in rural cancer care, which had not previously been explored.

The use of a small convenience sample from each health region may have resulted in a homogenous sample, which may have caused data bias. Notably, two-thirds of the sample was male, and this may have influenced the findings, in particular because men may be more likely to delay seeking medical advice. ${ }^{33}$ As well, the RCNC role may have had a positive influence on some patients' cancer journeys. Furthermore, this study did not directly consider issues such as specific disparities in cancer outcomes for rural indigenous Australians and other minority groups.

This study did not explore the decision making of people with a diagnosis of cancer who lived in urban areas, and it is possible that some of the issues described by rural cancer patients would be mirrored by their urban counterparts. A study comparing and contrasting the experiences of rural and urban cancer patients would be useful to determine what issues are specific to rural populations.

\section{Implications for Practice}

The findings of this study suggest that rural people with symptoms that may lead to a cancer diagnosis need encouragement to seek help earlier from primary health care services. 
Rural cancer nurses could play an important role in assisting people to seek help and to support them immediately after diagnosis with information about treatment decisions and practical advice about issues such as travel and finances. Strategies that encourage and promote self-management for cancer patients navigating the health system may also be helpful to promote health literacy and empower cancer patients to seek options that suit their circumstances. Furthermore, the findings also support an approach that allows for early identification of opportunities for undertaking treatment closer to home where appropriate. This includes the expanded use of telehealth to link rural patients with specialists in tertiary centers when possible to avoid long trips and associated financial, travel, and accommodation difficulties experienced for short review appointments.

\section{References}

1. Australian Institute of Health and Welfare. Rural, Regional and Remote Health: A study on Mortality. 2nd ed. Canberra, Australia: AIHW; 2007.

2. Clinical Oncological Society of Australia. Mapping Rural and Regional Oncology Services in Australia. 2006. http://www.cosa.org.au/cosa/File/ publications/Mapping_regional_oncology_services_MAR06.pdf. Accessed July 6, 2010.

3. Jong K, Smith D, Yu X, O'Connell DL, Goldstein D, Armstrong BK. Remoteness of residence and survival from cancer in New South Wales. MJA. 2004;82(11):618-622.

4. Western Australian Cancer \& Palliative Care Network. Model of Care for Cancer. Perth: Department of Health, Western Australia; 2008.

5. Australian Institute of Health and Welfare and Australasian Association of Cancer Registries (AACR). Cancer Survival in Australia 1992-1997: Geographic Categories and Socioeconomic status. AIHW cat. no. CAN 17. Canberra, Australia: Australian Institute of Health and Welfare (cancer series no. 22); 2003.

6. Smith LK, Pope C, Botha JL. Patients' help-seeking behaviour and delay in cancer presentation: a qualitative synthesis. Lancet. 2005;366: 825-831.

7. Campbell NC, Elliott AM, Sharp L, Ritchie LD, Cassidy J, Little J. Rural and urban differences in stage at diagnosis of colorectal and lung cancers. Br J Cancer. 2001;84:910-914.

8. Launoy G, Le Coutour X, Gignoux M, Pottier D, Dugleux G. Influence of rural environment on diagnosis, treatment and prognosis of colorectal cancer. J Epidemiol Community Health. 1992;46:365-367.

9. Liff JM, Chow WH, Greenberg RS. Rural-urban differences in stage of diagnosis. Cancer. 1991;67:1454-1459.

10. Jiwa M, Halkett G, Aoun S, et al. Factors influencing the speed of cancer diagnosis in rural Western Australia: a general practice perspective. $B M C$ Fam Pract. 2007;8:27-33.

11. Hall S, Holman CD, Sheiner H, Hendrie D. The influence of socioeconomic and locational disadvantage on survival after a diagnosis of lung or breast cancer in Western Australia. $J$ Health Serv Res Policy. 2004;9(suppl 2):S2-S10.

12. Elliott TE, Elliot BA, Renier CM, Haller IV. Rural-urban differences in cancer care: results from the Lake Superior Rural Cancer Care Project. Minn Med. 2004;87(9):44-50.

13. Esnaola NF, Knott K, Finney C, Gebregziabher M, Ford ME. Urbanrural residence moderates effect of race on receipt of surgery in patients with nonmetastatic breast cancer: a report from the South Caroline central cancer registry. Ann Surg Oncol. 2008;15(7):1828-1236.

14. Kok DL, Chang JH, Erbas B, et al. Urban-rural differences in the management of screen-detected invasive breast cancer and ductal carcinoma in situ in Victoria. Aust N Z J Surg. 2006;76:996-1001.

15. Fitch MI, Gray RE, McGowan T, et al. Travelling for radiation cancer treatment: patient perspectives. Psychooncology. 2003;12:664-674.
16. Mathews M, West R, Buehler S. How important are out-of-pocket costs to rural patients' cancer care decisions? Can J Rural Med. 2009;14(2): 54-60.

17. Baume P. Radiation Oncology Inquiry: A Vision for Radiotherapy. Canberra, Australia: Commonwealth of Australia; 2002. http://www.health.gov.au/ roi/inquiry/report.htm. Accessed July 6, 2010.

18. Hall SE, Holman CDJ, Threlfall T, et al. Lung cancer: an exploration of patient and general practitioner perspectives on the realities of care in rural Western Australia. Aust J Rural Health. 2008;16(6):355-362.

19. Hegney D, Pearce S, Rogers-Clark C, et al. Close, but still too far: the experience of Australian people with cancer commuting from a regional to a capital city for radiotherapy treatment. Eur J Cancer Care. 2005;14(1): 75-82.

20. Stebbins RA. Exploratory Research in the Social Sciences. Thousand Oaks, CA: A Sage University Paper; 2001

21. Australian Institute of Health and Welfare. The Rural, Remote and Metropolitan Areas (RRMA) Classification. http://www.aihw.gov.au/ruralhealth/ remotenessclassifications/rrma.cfm. Accessed July 21, 2010.

22. Threlfall TJ, Thompson JR. Cancer Incidence and Mortality in Western Australia, 2007. Perth, Western Australia: Department of Health, Western Australia; 2009. Statistical series no. 86.

23. Salyes H, Belli RF, Serrano E. Interviewer variance between event history calendar and conventional questionnaire interviews. Public Opin Q. 2010; 74(1):140-153

24. van der Vaart W, Glasner T. Applying a timeline as a recall aid in a telephone survey: a record check study. Appl Cognit Psychol. 2007;21: 227-238.

25. Elo $\mathrm{S}$, Kyngas $\mathrm{H}$. The qualitative content analysis process. $J$ Adv Nurs. 2008;62(1):107-115.

26. Burla L, Knierim B, Barth J, et al. From text to codings: intercoder reliability assessment in qualitative content analysis. Nurs Res. 2008;57(2): 113-117.

27. Emden C, Sandelowski M. The good, the bad and the relative, part one: conceptions of goodness in qualitative research. Int J Nurs Pract. 2002; 4(4):206-212.

28. Kirsch SED, Brandt PA. Telephone Interviewing: a method to reach fathers in family research. J Fam Nurs. 2002;8(1):73-84.

29. Musselwhite K, Cuff L, McGregor L, King KM. The telephone interview is an effective method of data collection in clinical nursing research: a discussion paper. Int J Nurs Stud. 2007;44:1064-1070.

30. Harris R, Kelly D, Hunt JA, et al. Accessing elite nurses for research: reflections on the theoretical and practical issues of telephone interviewing. J Res Nurs. 2008;13:236-248.

31. Garbett R, McCormack B. The experience of practice development: an exploratory telephone interview study. J Clin Nurs. 2001;10:94-102.

32. O'Kane G, Craig P, Sutherland D. Riverina men's study: an exploration of men living in rural area's attitudes to health and body image. Nutr Diet. 2008;65:66-71.

33. Buckley D, Lower T. Factors influencing the utilisation of health services by men living in rural areas. Aust Health Rev. 2002;25(2):11-15.

34. Howat A, Veitch C, Cairns W. A descriptive study comparing health attitudes of urban and rural oncology patients. Rural Remote Health. 2006;6:563. http://www.rrh.deakin.edu.au. Accessed July 6, 2010.

35. Powe BD, Weinrich $S$. An intervention to decrease cancer fatalism among rural elders. Oncol Nurs Forum. 1999;26(3):583-588.

36. Lamarche PA, Pineault R, Haggerty J, Hamel M, Levesque J, Gautier J. The experience of primary health care users: a rural-urban paradox. Can J Rural Med. 2010;15(2):61-67.

37. Beatty S, Stevens W, Stevens G, Kolbe J, Cox B. Lung cancer patients in New Zealand initially present to secondary care through the emergency department rather than by referral to a respiratory specialist. $N Z$ Med J. 2009;122(1294):33-41.

38. Clavarino AM, Lowe JB, Carmont S, Balanda K. The needs of cancer patients and their families from rural and remote areas of Queensland. Aust J Rural Health. 2002;10(4):188-195.

39. White NJ, Given BA, Devoss DN. The advanced practice nurse: meeting the information needs of the rural cancer patient. J Cancer Educ. 1996; 11(4):203-209. 
40. Watson AC. The role of the psychosocial oncology CNS in a rural cancer outreach program. Clin Nurse Spec. 1993;7(5):259-265.

41. Smith SM, Campbell NC. Provision of oncology services in remote rural areas: a Scottish perspective. Eur J Cancer Care. 2004;13: 185-192.

42. McKenna H, McCann S, McCaughan E, Keeney S. The role of an outreach oncology nurse practitioner: a case study evaluation. Eur $J$ Oncol Nurs. 2004;8:66-77.

43. Drury VB, Inma C. Exploring patient experiences of cancer services in regional Australia. Cancer Nurs. 2010;33(1):25-31.

44. Eley RM, Rogers-Clark C, Murray K. The value of a breast care nurse in supporting rural and remote cancer patients in Queensland. Cancer Nurs. 2008;31(6):10-18.

45. Koutsopoulou S, Papathanassoglou EDE, Katapodi MC, Patiraki EI. A critical review of the evidence for nurses as information providers to cancer patients. J Clin Nurs. 2010;19:749-745.
46. WA Country Health Service. Rural Cancer Nurse Coordinator ServiceInformation for People Affected by Cancer. Perth, Western Australia: Department of Health; 2010.

47. Clayton JM, Butow PN, Tattersall MHN, et al. Randomized controlled trial of a prompt list to help advanced cancer patients and their caregivers to ask questions about prognosis and end-of-life care. J Clin Oncol. 2007;25(6):715-723.

48. Singh R, Mathiassen L, Stachura ME, Astapova EV. Sustainable rural telehealth innovation: a public health case study. Health Serv Res. 2010; 45(4):985-1004.

49. Olver I, Selva-Nayagam S. Evaluation of a telemedicine link between Darwin and Adelaide to facilitate cancer management. Telemed J. 2000; 6(2):213-218.

50. Kunkler IH, Prescott RJ, Lee RJ, et al. TELEMAM: a cluster randomised trial to assess the use of telemedicine in multi-disciplinary breast cancer decision making. Eur J Cancer. 2007;43:506-2514.

\section{Appendix A • Semistructured Interview Guide}

Purpose: Semistructured interviews are being used for qualitative data relating to the study. Interviews will be used to gain information about the cancer journey of the participant and reasons for the decisions they have made about their treatment.

Participants: Interviews will be conducted with rural people with cancer diagnosis in 2008-2009.

Time: It is expected that the interview will last approximately 45 to 60 minute.

\section{Introduction}

Little is known about how rural people with cancer make decisions regarding their cancer. We would like to speak with you today about aspects of your cancer journey including when you were diagnosed and what decisions you have made about cancer treatments. We will be using a calendar marked with important dates to help jog your memory about when different things happened.

\section{Guiding Questions}

1. Can you describe to me what symptoms made you first think that something was wrong?

\section{Prompts:}

a. Describe these symptoms

b. What made you think these symptoms were unusual/suggestive?

c. Using the landmarking calendar, can you recall when you first experienced the symptoms?

d. What did you do about these symptoms?

2. Can you describe to me what made you decide to see your doctor?

\section{Prompts:}

a. Was there a delay in between experiencing the symptoms and deciding that you should see the doctor?

b. Using the landmarking calendar, can you recall when you first made the decision to see your doctor?

c. What stopped you/delayed you in making this decision?

d. What helped you to make this decision?

3. Did you make an appointment to see the doctor directly after you made the decision to do so?

\section{Prompts:}

a. Was there a delay in between making the decision to see your doctor and booking an appointment?

b. Using the landmarking calendar, can you recall when you first made an appointment to see your doctor?

c. What stopped/delayed you in making this decision?

d. What helped you to make this decision?

\section{Making an appointment}

\section{Prompts:}

a. Was there a delay in between booking an appointment and actually seeing your doctor?

b. Using the landmarking calendar, can you recall when you first saw your doctor?

c. What stopped/delayed you from seeing your doctor?

d. What helped you to see your doctor? 


\section{Appendix A • Semistructured Interview Guide, continued}

5. Following the appointment: investigations

\section{Prompts:}

a. What investigations (eg, $\mathrm{x}$-rays blood test, computed tomography/positron emission tomography scans) were prescribed by your doctor?

b. Did you follow the recommendations of your medical practitioner?

c. Why? Why not?

d. Using the landmarking calendar, can you recall when you undertook your first investigations?

e. What stopped/delayed you from undertaking investigations?

f. What helped you to undertake investigations?

6. Following the appointment: referrals

\section{Prompts:}

a. What referrals were prescribed by your doctor?

b. Did you follow the recommendations of your medical practitioner?

c. Why? Why not?

d. Using the landmarking calendar, can you recall when you saw the specialist?

e. What stopped/delayed you from seeing a specialist?

f. What helped you to see a specialist?

7. Following the appointment: treatment

We want to know about what treatment you have had and what decisions you had to make about your treatment.

\section{Prompts:}

a. What treatments were prescribed by your doctor/specialist?

b. Did you follow the recommendations of your medical practitioners?

c. Why? Why not?

d. Using the landmarking calendar, can you recall when you undertook your first treatment?

e. Where did you have your treatment? Why did you choose to have it in this location? Did you have any other options? What were they?

f. What stopped you from receiving treatment or made treatment difficult?

g. What helped you or made it easier to have treatment?

h. Are you happy with the decisions you made about your treatment?

i. Did you need more help making decisions about your treatment? What would have helped?

Do you have any other questions or comments? 